Revue internationale P.M.E.

Économie et gestion de la petite et moyenne entreprise

\title{
Repérage de la fonction des ressources humaines en P.M.E.
}

\section{Henri Mahe de Boislandelle}

Volume 3, numéro 1, 1990

URI : https://id.erudit.org/iderudit/1007944ar

DOI : https://doi.org/10.7202/1007944ar

Aller au sommaire du numéro

Éditeur(s)

Presses de l’Université du Québec

ISSN

0776-5436 (imprimé)

1918-9699 (numérique)

Découvrir la revue

Citer cet article

Mahe de Boislandelle, H. (1990). Repérage de la fonction des ressources humaines en P.M.E. Revue internationale P.M.E., 3(1), 27-37.

https://doi.org/10.7202/1007944ar

\section{Résumé de l'article}

Cet article propose d'analyser la gestion des Ressources Humaines en P.M.E. en distinguant plusieurs niveaux d'approche :

- celui de l'administration du personnel (niveau I)

- celui des politiques de personnel (niveau II)

- et celui de l'organisation générale, associée au style de direction, comme déterminants (niveau III).

Cette démarcation en niveaux se révèle éclairante de la diversité des pratiques en fonction de la taille des entreprises et du poids accordé par les dirigeants aux Ressources Humaines.

En effet, la formalisation de la fonction étant souvent faible dans les entreprises de petite dimension, seul le repérage des tâches et des acteurs permet d'identifier la nature et l'importance exacte de celle-ci.

Considérant sous cet angle une étude empirique française récente, il permet de mieux comprendre la réalité de la fonction et ses contingences. 


\title{
Repérage de la fonction des ressources humaines en P.M.E.
}

\author{
Henri MAHE de BOISLANDELLE' \\ Université de St-Étienne
}

\begin{abstract}
RÉSUME
Cet article propose d'analyser la gestion des Ressources Humaines en P.M.E. en distinguant plusieurs niveaux d'approche :

- celui de l'administration du personnel (niveau I)

- celui des politiques de personnel (niveau II)

- et celui de l'organisation générale, associée au style de direction, comme déterminants (niveau III).

Cette démarcation en niveaux se révèle éclairante de la diversité des pratiques en fonction de la taille des entreprises et du poids accordé par les dirigeants aux Ressources Humaines.

En effet, la formalisation de la fonction étant souvent faible dans les entreprises de petite dimension, seul le repérage des tâches et des acteurs permet d'identifier la nature et l'importance exacte de celle-ci.

Considérant sous cet angle une étude empirique française récente, il permet de mieux comprendre la réalité de la fonction et ses contingences.
\end{abstract}

\section{ABSTRACT}

This paper proposes a multi-level approach for analyzing human resources management in small and medium-sized firms :

- personnel administration (level 1)

- personnel policies (level 11)

- general organization associated with management style (level 111)

This division into levels reveals the wide range of practices depending on the size of the firms and the importance accorded to human resources by managers.

In smaller firms, human resources management is often carried out in an less formal way, and therefore its exact nature and importance can only be discerned by identifying tasks and actors.

The paper, by examining a recent French empirical research study from this point of view, about a better understanding of the function and its requirements.

* Henri Mahé de Boislandelle est professeur à l'Université de Saint-Etienne où il enseigne la gestion des ressources humaines. Il anime une équipe de recherche composée de praticiens et de chercheurs en GRH. Adresse : CEGERH, département GEA/UT, 99, ave. d'Occitanie, 34075 Montpellier, France. 


\section{RESUMEN}

En este articulo se propone el analisis de la gestion de los Recursos Humanos en las PyME, a través de la distincion de los siguientes niveles de planteamiento:

- el de la administracion del personal (nivel 1)

- el de las politicas de personal (nivel 11)

- y el de la organizacion general, asociada al estilo de direccion como factores determinantes (nivel 111).

Esta delimitacion de niveles es reveladora en cuanto a la diversidad de practicas en funcion de la dimension de las empresas y del peso que acuerdan los dirigentes a los Recursos Humanos.

En efecto, la formalizacion de la funcion siendo a menudo débil dentro de las empresas de pequena dimension, solo el reconocimiento de las tareas y de los actores permite de identificar la indole y la importancia exacta de estas ultimas.

Considerando bajo este angulo un estudio empirico francés reciente, es posible de comprender mucho mejor la realidad de la funcion y sus contingencias. 


\section{Introduction}

Si gestionnaires et économistes s'accordent aujourd'hui à reconnaître l'intérêt et l'importance des petites et des moyennes entreprises, quelques-uns seulement s'emploient à en souligner la spécificité d'approche (Julien et Marchesnay, 1988). Séduits par l'idée de spécificité, force est de reconnaître qu'ils leur est parfois difficile de la mettre en lumière, tant les instruments d'analyse dominants sont imprégnés par ceux utilisés dans les grandes organisations.

Engagés dans l'étude de la Gestion des ressources humaines (G.R.H.) en P.M.E., nous sommes amenés à partager cette conviction car nous avons été conduits pour cela à adopter un mode d'investigation particulier sans lequel le caractère contingent de cette fonction dans ce type d'entreprises nous aurait probablement échappé.

Désireux, en effet, d'analyser la fonction ressources humaines dans les P.M.E., c'est-à-dire dans des entreprises ayant des activités et des tailles disparates (de 1 à 500 salariés), il nous apparaît rapidement nécessaire de différencier service et fonction; car, si la fonction existe toujours, comme nous le montrons plus loin, en revanche la structuration en service de personnel relativement autonome n'apparaît qu'à partir d'une certaine catégorie de taille et de façon non systématique.

Confrontés par conséquent à l'identification d'une fonction, peu ou incomplètement apparente sur un plan formel, il nous est utile d'inverser les termes habituels de l'investigation, c'est-à-dire de définir d'abord un contenu et de remonter ensuite vers un contenant structurel plus ou moins clairement formalisé.

Partant de là, nous adopterons une définition de la G.R.H. ou, plus précisément, nous en indiquerons les différents niveaux d'acceptation afin de procéder ensuite à un questionnement. L'objet de celui-ci sera de savoir : qui fait quoi en G.R.H. dans les P.M.E. ?

Nous référant alors à ces instruments conceptuels et méthodologiques, nous présenterons quelques résultats d'enquêtes réalisées en France sur des échantillons régionaux et nous essaierons d'en tirer quelques pistes d'approfondissement pour de nouvelles recherches.

\section{Quel contenu pour quel contenant ?}

La confusion ou l'attribution dans l'esprit du dirigeant d'une grande partie de la G.R.H. avec la direction générale de l'entreprise explique en grande partie la désignation plus ou moins étroite d'un responsable ou d'un service, ou encore, la répartition de différentes tâches sur plusieurs personnes internes ou externes à l'entreprise. Il apparaît que la différenciation Direction Générale/G.R.H. s'accentue avec la taille de l'entreprise, c'est-à-dire en même temps que la charge des problèmes de personnel se précise ou exige d'être explicités. Pour montrer ce processus, il 
importe de considérer et de dénombrer l'ensemble des actions susceptibles de concerner la G.R.H. dans ses aspects les plus généraux jusque dans ses aspects les plus administratifs.

Nous proposons pour cela de distinguer trois niveaux : celui de l'administration du personnel, celui des politiques de personnel et celui de l'organisation et de la direction générale comme cadre et contexte des Ressources Humaines.

La démarcation entre ces niveaux se révèle éclairante des pratiques dans telle ou telle entreprise. Elle permet, en effet, de situer la G.R.H. par rapport à trois niveaux qui se confondent parfois avec les visions de la direction.

Ces visions peuvent également être interprêtées comme moyens de mesurer à la fois l'ambition et la délégation de responsabilité en G.R.H..

\section{Niveau I, vision I}

A ce niveau, les personnes en charge du personnel ont à effectuer une série de tâches d'exécution codifiées et précises selon des échéanciers, à appliquer des règles juridiques et des directives émanant du dirigeant de l'entreprise.

Si ce niveau se confond avec la vision de la G.R.H. de l'entreprise, cela signifie que le dirigeant a une conception réductrice de la fonction qu'il ramène à de la simple administration du personnel. Il se réserve en revanche, dans ce cas là, l'ensemble des décisions relevant du niveau suivant qu'il assimile à de la direction générale.

\section{Niveau II, vision II}

Ce niveau conceme celui des politiques de personnel, c'est-à-dire des choix effectués par le dirigeant (ou par un responsable délégué à partir d'une certaine dimension).

Ces choix concernent l'emploi, la rémunération, les conditions de travail, la formation, les relations sociales...

Le niveau II fait l'objet de délégation partielle et sélective en P.M.E..

Si ce niveau correspond à la vision de la G.R.H. par le dirigeant, cela signifie qu'il dispose d'une vision enrichie de la G.R.H. conforme à l'acceptation couramment admise aujourd'hui et qu'il est à même de distinguer les niveaux I et II.

\section{Niveau III, vision III}

Au-delà des domaines précédents, on peut considérer la structure de l'organisation sur un plan général ou fonctionnel, le style de commandement, les modes participatifs... comme les déterminants de la G.R.H. dont ils constituent le contexte. 
Ici la fonction dépasse le cadre de toute délégation sur un responsable de personnel puisqu'elle concerne tout le champ de l'espace relationnel de l'entreprise.

Cette vision hétérodoxe, car traditionnellement du domaine managérial, mérite, à nos yeux, d'être prise en compte au plan de la G.R.H..

Il apparaît en effet aujourd'hui utile de considérer la relation dialectique dirigeant/personnel et plus généralement encadrement/personnel comme au coeur de la G.R.H. notamment sur le plan de la politique de communication et de participation. Différentes publications à succès parues ces dernières années témoignent de ces nouvelles préoccupations (Prix de l'Excellence, Entreprise du $3^{\circ}$ type...).

Souvent domaine réservé du dirigeant, ce niveau n'en est pas moins capital à appréhender dans une identification de fonction. S'il est accepté comme partie intégrante de la G.R.H. par le dirigeant, il traduit l'expression d'une vision ambitieuse et décloisonnée propice à des remises en cause susceptibles d'affecter aussi les autres niveaux.

De cette troisième optique, on en tire assez naturellement la conclusion que la G.R.H. ne se réduit pas uniquement à la gestion du personnel mais que la place du dirigeant étant fondamentale, il sera nécessaire de l'inclure dans l'analyse. On le considérera alors comme objet et sujet de gestion.

Partant de ce modèle de contenu en trois niveaux, considérons maintenant la réalité de la fonction R.H. dans les P.M.E. en nous appuyant sur un ensemble d'observations de terrain.

\section{2 Étude empirique de la fonction}

La fonction personnel en P.M.E. a fait l'objet en France de quelques rares études empiriques. Parmi celles-ci, l'étude montpellièraine que nous avons conduite nous semble la plus approfondie. Nous nous limiterons donc à sa présentation en considérant d'une part la méthodologie et la problématique et, d'autre part, les principaux résultats.

\subsection{Méthodologie et problématique}

Réalisée sur quatre années successives ${ }^{1}$ à partir d'échantillons complémentaires et une méthodologie constante, l'étude concerne au total 160 P.E. et P.M.E./ P.M.I. du Languedoc-Roussillon et du Midi-Pyrénées réparties en quatre catégories de taille : de 1 à 9 salariés (I), de 10 à 49 salariés (II), de 50 à 199 salariés (III) et 200 à 499 salariés (IV). Les appellations retenues étant respectivement T.P.E. (très petite

1 Enquêtes réalisées en 84, 85, 86, 87 et 89 à Montpellier par le C.E.G.E.R.H. 
entreprise), P.E. (petite entreprise), M.E. (moyenne entreprise) et M.G.E. (moyenne grande entreprise). Les échantillons retenus sont distribués à peu près également par catégories de tailles. Les questionnaires administrés sous forme d'entretien avec les dirigeants comportent une majorité de questions fermées et quelques-unes semiouvertes.

Parmi les nombreuses interrogations sous-jacentes à cette investigation, les principales questions étaient de savoir :

- qui assure la G.R.H. en P.E. et P.M.E./P.M.I. ?

- ou, plus précisément : qui fait quoi en la matière?

en distinguant :

- d'une part, la prise en charge interne par : le dirigeant, un responsable éventuel du personnel, un responsable administratif ou un comptable;

- et, d'autre part, la prise en charge partielle externe par : un expert comptable, un conseiller juridique et/ou une société de service et de conseil en informatique (SSCI).

Le modèle implicite de questionnement qui a servi de fond au questionnaire s'est appuyé sur les visions I et II que nous avons définies précédemment. La vision III que nous ne faisions que pressentir à l'époque a été traitée hâtivement ${ }^{2}$.

Préoccupés notamment de connaître le poids accordé par la direction de l'entreprise aux tâches d'administration et de politiques du personnel, nous avons «noté» l'intérêt et l'importance de l'ensemble des domaines et celà pour l'ensemble des tailles.

Les enquêtes réalisées au fil des années ont été de plus en plus affinées et précises (nous avons à chaque fois tenu compte de l'imprécision et des oublis des questionnaires antérieurs).

Pour compléter et améliorer le questionnaire et augmenter la fiabilité des informations recueillies, nous avons eu recours à des entretiens avec d'autres personnes de l'entreprise que le seul dirigeant.

La synthèse des résultats que nous présentons ici nous semble résumer assez bien le panorama de la G.R.H. en P.E. et P.M.E. pour le sud de la France.

\section{$2.2 \quad$ Résultats et commentaires}

Afin de rendre compte de l'essentiel, nous avons regroupé l'ensemble des résultats d'enquêtes dans un tableau récapitulatif (Mahé de Boislandelle, 1988).

2 Dans d'autres enquêtes effectuées par nous-mêmes sur «La formation des dirigeants de petite entreprise» nous y avions partiellement remédié (c.f. «La formation des dirigeants en période de démarrage», Revue Enseignement et Gestion, 1985). 
Le premier concerne le niveau de l'administration et le second celui des politiques. Leur structure de présentation est la suivante :

- en ligne sont distinguées les opérations par nature numérotées de 1 à 16 et de 8 à 15; les numéros 7 et 16 sont respectivement destinés à éclairer sur les modalités de prise en charge informatique et sur le caractère prévisionnel de la gestion du personnel.

- en colonne apparaissent successivement :

$\checkmark$ le poids accordé par la direction (noté par des étoiles allant de 0 à XXX)

$\checkmark$ les acteurs de la prise en charge dans et hors de l'entreprise :

- dans l'entreprise ont été distingués le dirigeant, le responsable du personnel, un administratif ou un comptable,

- hors de l'entreprise sont considérés les sous-traitants que sont les conseillers extérieurs, experts comptables et S.S.C.I.

Les notations adoptées $(-, 1,2$ ou 3$)$ figurent l'ordre de prise en charge (il s'agit d'une notation ordinale). Lorsque (c) apparaît, cela signifie qu'un recours au conseil est parfois utilisé.

La dernière ligne est relative à la fréquence de la prise en charge entre les différentes catégories d'acteurs. Pour une catégorie de taille le total doit être de $100 \%$.

On peut tirer, pour l'essentiel, de la lecture du tableau que la taille influence le poids des différentes variables et leur prise en charge aussi bien au plan de l'administration du personnel que des politiques de personnel.

\section{L'administration du personnel (vision I)}

Certaines tâches voient leur poids s'accroître avec leur taille (ex. : mise en application des dispositions légales, recours au traitement informatique...), d'autres, leur poids diminuer car elles semblent mieux maîtrisées (ex. : calcul des salaires et des charges sociales).

Quelle que soit la taille, on observe rarement une prise en charge par le dirigeant de la plupart des tâches. Bien qu'en proportion minoritaire (statistiquement parlant), les dirigeants s'y impliquent (s'en occupent) pour les tailles I et II.

Par ordre d'importance décroissante de la prise en charge, on note pour la taille I : un comptable externe, le dirigeant, un administratif (secrétaire) ou comptable interne. Dans les P.E. familiales, il s'agit souvent du conjoint (ou d'un proche).

Avec l'accroissement de la taille, le dirigeant a tendance à s'effacer au profit du comptable ou d'un administratif (tailles II et III). Dans la taille IV on relève assez fréquemment l'apparition d'un responsable du personnel (65\%). A y regarder de plus près, celui-ci n'assure pas cependant en priorité la totalité des charges (par exemple les salaires et leur traitement informatique lui sont rarement réservés). 
Tab. 1. Comparaison des modes d'administration du personnel pour les quatre catégories de taille

\begin{tabular}{|c|c|c|c|c|c|c|c|c|c|c|c|c|c|c|c|c|c|c|c|c|}
\hline \multirow{4}{*}{$\begin{array}{l}\text { Nature } \\
\text { des opérations }\end{array}$} & & & & & \multicolumn{16}{|c|}{ Acteurs de la prise en charge } \\
\hline & & & & & & & & & & Jans & itrep & & & & & & & rs de & l'entr & \\
\hline & \multicolumn{4}{|c|}{$\begin{array}{l}\text { Poids accordé } \\
\text { par la direction }\end{array}$} & \multicolumn{4}{|c|}{ Dirigeant } & \multicolumn{4}{|c|}{$\begin{array}{l}\text { Responsable } \\
\text { du personnel }\end{array}$} & \multicolumn{4}{|c|}{$\begin{array}{l}\text { Administratif, } \\
\text { adjoint, comptable }\end{array}$} & \multicolumn{4}{|c|}{$\begin{array}{l}\text { Conseil extérieur, } \\
\text { SSCl... }\end{array}$} \\
\hline & 1 & $\|$ & III & IV & 1 & ॥ & III & IV & 1 & $\|$ & III & IV & 1 & $\|$ & IIII & IV & 1 & $\|$ & III & IV \\
\hline 1. Tenue de fichiers & $x$ & $x$ & $x$ & $x x$ & 2 & 2 & - & - & - & - & - & 1 & 3 & 1 & 1 & 2 & 1 & 3 & 2 & $\cdot$ \\
\hline $\begin{array}{l}\text { 2. Déclarations légales } \\
\text { diverses }\end{array}$ & $x x$ & $x x$ & $x$ & $x x$ & 2 & 2 & - & - & - & - & - & 1 & 3 & 1 & 1 & 2 & 1 & 3 & 2 & - \\
\hline $\begin{array}{l}\text { 3. Relations avec les ser- } \\
\text { vices publics du travail } \\
\text { et de l'emploi }\end{array}$ & $x$ & $x x$ & $x x$ & $x X$ & 2 & 2 & 2 & 3 & - & - & - & 1 & 3 & 1 & 1 & 2 & 1 & 3 & 3 & $\cdot$ \\
\hline $\begin{array}{l}\text { 4. Calcul des salaires ot } \\
\text { des charges sociales }\end{array}$ & $X X X$ & $X X X$ & $x x$ & $x X$ & 2 & 3 & - & 3 & - & - & - & 2 & 3 & 1 & 1 & 1 & 1 & 2 & 2 & c \\
\hline $\begin{array}{l}\text { 5. Information sur la lé- } \\
\text { gislation du travail et } \\
\text { de la Sécurité sociale,.. }\end{array}$ & $x$ & $X X$ & $x x$ & $x x$ & 2 & 3 & 3 & 3 & - & - & - & 1 & 3 & 1 & 1 & 2 & 1 & 2 & 2 & c \\
\hline $\begin{array}{l}\text { 6. Mise en application des } \\
\text { dispositions légales (sé- } \\
\text { curité, ACT, BS (2)..) }\end{array}$ & $x$ & $x x$ & $x x x$ & $x x x$ & 1 & 2 & 1 & - & - & - & - & 1 & 2 & 1 & 2 & 2 & - & - & 3 & $3 c$ \\
\hline $\begin{array}{l}\text { 7. Recours au traitement } \\
\text { formatique (paie, fi- } \\
\text { chiers, ...) }\end{array}$ & $x$ & $x x$ & $x x$ & $x x$ & 3 & 3 & - & - & - & - & - & 3 & 2 & 1 & 1 & 1 & 1 & 2 & 2 & 2 \\
\hline $\begin{array}{l}\text { Fréquence de la prise en cha } \\
\text { les différentes catégories d'ac }\end{array}$ & & & & & $30 \%$ & $15 \%$ & $20 \%$ & $5 \%$ & - & - & - & $65 \%$ & $20 \%$ & $55 \%$ & $65 \%$ & $30 \%$ & $50 \%$ & $30 \%$ & $15 \%$ & - \\
\hline
\end{tabular}

(1) Dans la taille III l'aadministratifo chargé d'administrer le personnel est parfois qualifié de responsable du personnel même si son activité, à ce titre, n'est qu'à temps partiel.

(2) BS = Bilan social.

$C=$ Conseil 
Tab. 1 (suite). Comparalson des modes d'administration du personnel pour les quatre catégorles de taille

\begin{tabular}{|c|c|c|c|c|c|c|c|c|c|c|c|c|c|c|c|c|c|c|c|c|}
\hline \multirow{4}{*}{$\begin{array}{l}\text { Nature } \\
\text { des opérations }\end{array}$} & & & & & \multicolumn{16}{|c|}{ Acteurs de la prise en charge } \\
\hline & & & & & \multicolumn{12}{|c|}{ Dans l'entreprise } & \multirow{2}{*}{\multicolumn{4}{|c|}{$\begin{array}{c}\text { Hors de l'entreprise } \\
\text { Conseil extérieur, } \\
\text { SSCl... }\end{array}$}} \\
\hline & \multicolumn{4}{|c|}{$\begin{array}{l}\text { Poids accordé } \\
\text { par la direction }\end{array}$} & \multicolumn{4}{|c|}{ Dirigeant } & \multicolumn{4}{|c|}{$\begin{array}{l}\text { Responsable } \\
\text { du personnel }\end{array}$} & \multicolumn{4}{|c|}{$\begin{array}{l}\text { Administratif, } \\
\text { adjoint, comptable }\end{array}$} & & & & \\
\hline & 1 & $\|$ & III & IV & 1 & ॥ & III & IV & 1 & ॥ & III & IV & 1 & $\|$ & III & IV & 1 & $\|$ & III & IV \\
\hline $\begin{array}{l}\text { 8. Temps de travail et ho- } \\
\text { raires }\end{array}$ & $x$ & $x$ & $x x$ & $X X(X)$ & 1 & 1 & - & 1 & - & - & - & 2 & - & - & - & 3 & - & - & - & c \\
\hline 9. Modes de rémunération & $x$ & $x x$ & $x x$ & $x X$ & 1 & 1 & - & 1 & - & - & - & 2 & $2 c$ & $2 c$ & - & 3 & - & $2 c$ & - & c \\
\hline $\begin{array}{l}\text { 10. Évaluation du personnel, } \\
\text { promotion, gestion des } \\
\text { carrières }\end{array}$ & $\begin{array}{c}x \\
\text { ou } \\
0\end{array}$ & $\begin{array}{c}x \\
\text { ou } \\
0\end{array}$ & $x X$ & $x X X$ & 1 & 1 & - & 3 & - & - & - & 1 & - & - & - & 2 & - & - & - & c \\
\hline 11. Politique sociale & - & - & $\stackrel{0 a ̀}{x}$ & $x$ & 1 & 1 & 1 & 1 & - & - & - & 2 & - & - & 2 & 3 & - & - & - & - \\
\hline $\begin{array}{l}\text { 12. Définition et organisa- } \\
\text { tion du travail }\end{array}$ & $x$ & $x$ & $x x$ & $x x$ & 1 & 1 & 2 & 2 & - & - & - & 3 & $2 c$ & $2 c$ & 1 & 1 & - & - & $3 c$ & c \\
\hline $\begin{array}{l}\text { 13. Emploi (recrutement, rota- } \\
\text { tion, licenciement ...) }\end{array}$ & $x x x$ & $x x x$ & $x x x$ & $x x x$ & 1 & 1 & 1 & 1 & - & - & - & 2 & $2 c$ & $2 c$ & $2 c$ & $3 c$ & - & $3 c$ & 3 & c \\
\hline 14. Formation & - & $\begin{array}{c}x \\
\text { ou } 0\end{array}$ & $x x$ & $X X(X)$ & 1 & 1 & 2 & 3 & - & - & - & 1 & - & - & 1 & 2 & & 2c & $3 c$ & c \\
\hline $\begin{array}{l}\text { 15. ACT, relations sociales, } \\
\text { communication, parti- } \\
\text { cipation }\end{array}$ & 0 & $x$ & $x x$ & $x x$ & 1 & 1 & 1 & 2 & - & - & - & 1 & - & - & 2 & 3 & - & - & c & c \\
\hline $\begin{array}{l}\text { 16. Caractibre prévisionnel } \\
\text { de la gestion du person- } \\
\text { nel (plan à MT...) }\end{array}$ & 0 & $x$ & $x(x)$ & $\begin{array}{c}x \\
a \\
x x\end{array}$ & 1 & 1 & 2 & - & - & - & - & 1 & - & - & 1 & 2 & - & - & - & $3 c$ \\
\hline $\begin{array}{l}\text { "Fréquence de la prise en char } \\
\text { les différenies carégories d'acte }\end{array}$ & itre & & & & $100 \%$ & $100 \%$ & $70 \%$ & $40 \%$ & - & - & - & $40 \%$ & - & - & $30 \%$ & $20 \%$ & & & & \\
\hline
\end{tabular}

Légende: $c=$ conseil 
Concernant le recours au conseil ou à la sous-traitance externe, on observe globalement une tendance à l'effacement avec la taille (dans l'échantillon étudié, les entreprises procèdent à une internalisation des tâches).

\section{Les politiques de personnel (vision II)}

Contrairement à l'administration du personnel pour laquelle on peut observer des évolutions de sens opposé (poids croissant ou décroissant) suivant la taille, ici tous les domaines des politiques de personnel prennent du poids avec la dimension.

Les domaines où cela est le plus fortement marqué sont :

- les politiques de temps et horaires de travail,

- les politiques d'évaluation et de promotion,

- les politiques de formation,

- et, globalement, l'attitude prévisionnelle à l'égard de la G.R.H..

Dans d'autres domaines, à partir des tailles II ou III, l'importance se stabilise (ex. : les politiques de rémunération, l'organisation du travail, les relations sociales, etc.).

Dans tous les cas, quelle que soit la taille, la politique d'emploi (le recrutement notamment) revêt une importance tout à fait capitale. Contrairement à l'administration du personnel qui faisait apparaitre un effacement progressif du dirigeant, ici, en revanche, sa place reste globalement prépondérante. Ceci signifie que la délégation est absente de certains domaines et en tout cas partielle et sélective sur quelques autres.

Pour les tailles I et II, le dirigeant assume et assure tous les aspects des politiques du personnel sans partage.

A partir de la taille III (seuil de 50 salariés), on peut percevoir certaines délégations en matière d'organisation du travail, de politique de formation et parfois de réflexion prévisionnelle. Ces délégations s'effectuent au bénéfice d'un début d'encadrement (technique ou administratif).

Avec la taille IV, les délégations se précisent davantage et certaines formalisations s'affirment. Le responsable du personnel ( $40 \%$ des cas) ou un responsable à temps partiel (20\%) est associé à certains domaines des politiques dont il se voit confier en grande partie ou en totalité la conception et la mise en oeuvre (ex. : l'évaluation et les promotions du personnel, la formation, les relations sociales et la gestion prévisionnelle du personnel...). L'organisation du travail est confiée à un responsable technique (opérationnel). Le dirigeant conserve néanmoins la haute main sur une série de domaines (même s'il délègue partiellement) qu'il juge prépondérants : la politique du temps de travail et d'aménagement horaire, la 
politique de rémunération, la politique sociale, la politique d'emploi (notamment le recrutement et le licenciement).

De cette série d'enquêtes on retiendra une très grande diversité de situations de prises en compte de la G.R.H. que les statistiques globales ont tendance à gommer puisqu'elles confondent la multitude d'activités et de structures organisationnelles. Néanmoins un certain nombre de dominantes dans les comportements au niveau de la taille ont été soulignés.

\section{Conclusion}

Que tirer de l'analyse de contenu de la fonction et de l'étude empirique ?

D'abord, que la G.R.H. dans les quatre tailles de P.E. et P.M.E. que nous avons considérées :

- commence à apparaître comme une réalité multiple et contingente à la fois de la taille, du statut de l'entreprise et du type d'activité;

- que les dirigeants de P.E. et P.M.E. ont une philosophie des R.H. déterminante. Ils sont sensibles aux discours ambiants et sont très nettement influencés par le type de formation initiale qu'ils ont reçue;

- que la G.R.H., à l'heure actuelle, repose en P.E. et P.M.E. principalement sur la vision I et que la vision II gagne du terrain en fonction de la taille.

Ensuite, au plan des perspectives de recherche, qu'une importante étude globale, plus affinée sur de groséchantillons nationaux et internationaux, permettrait de corriger différentes imprécisions. Pareillement, la réalisation d'études spécialisées sur telles ou telles pratiques de gestion de l'emploi, de la rémunération, de la valorisation et de la participation ne pourrait qu'apporter de fructueux enseignements.

Enfin, une analyse sous l'angle de la vision III permettrait à n'en pas douter d'enrichir la réflexion, d'avoir de nouvelles propositions méthodologiques et des outils de gestion appropriés aux P.E. et P.M.E.. Ceci aiderait à aller plus loin que ce que nous avons tenté de faire dans notre ouvrage sur la «Gestion des Ressources Humaines dans les P.M.E.» (1988).

\section{BIBLIOGRAPHIE}

Julien, P.A. et Marchesnay,M., (1988), La petite entreprise, Paris, Vuibert-Gestion.

Mahé de Boislandelle, H., (1988), Gestion des Ressources Humaines dans les P.M.E., Paris, Economica, (notamment le modèle de mix-social). 